\title{
Efeitos da temperatura no ciclo de vida, exigências térmicas e estimativas do número de gerações anuais de Aedes aegypti (Diptera, Culicidae)
}

\author{
Eduardo B. Beserra ${ }^{1}$, Carlos R. M. Fernandes ${ }^{1}$, Suêide A. de O. Silva ${ }^{1}$, Lilian A. da Silva ${ }^{1}$ \& \\ José W. dos Santos ${ }^{2}$
}

1. Departamento de Biologia, Universidade Estadual da Paraíba, Rua Juvêncio Arruda s/n, Bodocongó, 58109-753 Campina Grande, PB, Brasil. (ebeserra@uol.com.br; ebarbosa@uepb.edu.br)

2. Centro Nacional de Pesquisa do Algodão, Embrapa Algodão, Rua Osvaldo Cruz, 1143, Centenário, 58107-720 Campina Grande, PB, Brasil. (jwsantos@cnpa.embrapa.br)

\begin{abstract}
Effects of temperature on life cicle, thermal exigency and number of generations per year estimation of Aedes aegypti (Diptera, Culicidae). The present work aims at estimating, based on a biological study, the thermal exigencies to the development and the number of generations per year of Aedes aegypti (Linnaeus, 1762) (Diptera, Culicidae) in field. The life cycle of the $A$. aegypti populations has been studied at constant temperatures of $18,22,26,28,32$ and $34 \pm 2^{\circ} \mathrm{C}$, and 12 hours photophase. The low threshold temperature of development $(\mathrm{Tb})$ and the thermal constant $(\mathrm{K})$ have been determined. The number of generations per year in laboratory and field has also been estimated. The favourable temperature to the A. aegypti development its between $22^{\circ} \mathrm{C}$ and $32^{\circ} \mathrm{C}$, and to the longevity and fecundity A. aegypti adult its between $22^{\circ} \mathrm{C}$ and $28^{\circ} \mathrm{C}$. The egg to adult basal temperature, thermal constant and the number of generations in field were, in order, $11.33 ; 8.99$ and $13.61{ }^{\circ} \mathrm{C}, 192.3 ; 213.2$ and 116.5 graus-days, and $23 ; 24.6$ and 30.3 generations to A. aegypti populations from Boqueirão (07 $\left.29^{\prime} 27^{\prime \prime} \mathrm{S}, 36^{\circ} 08^{\prime} 09^{\prime \prime} \mathrm{W}\right)$, Campina Grande $\left(07^{\circ} 13^{\prime} 32^{\prime \prime} \mathrm{S}, 35^{\circ} 54^{\prime} 15^{\prime \prime} \mathrm{W}\right)$ and Remígio (06 $\left.58^{\prime} 1^{\prime \prime} \mathrm{S}, 35^{\circ} 47^{\prime} 29^{\prime \prime} \mathrm{W}\right)$.
\end{abstract}

KEYWORDS. Insecta, Aedini, biology, thermal requeriments.

RESUMO. O trabalho teve por objetivo avaliar o efeito da temperatura sobre o ciclo de vida de Aedes aegypti (Linnaeus, 1762), determinar as exigências térmicas para o desenvolvimento e estimar o número de gerações anuais do inseto em campo. O ciclo de vida das populações de A. aegypti foi estudado nas temperaturas de $18,22,26,28,32$ e $34 \pm 2^{\circ} \mathrm{C}$ e fotofase de 12 horas, determinando-se ainda os limites térmicos inferiores de desenvolvimento $(\mathrm{Tb})$ e as constantes térmicas $(\mathrm{K})$ e estimado o número de gerações anuais do inseto em laboratório e em campo. A temperatura favorável ao desenvolvimento de A. aegypti encontra-se entre $22^{\circ} \mathrm{C}$ e $32^{\circ} \mathrm{C}$, e para a longevidade e fecundidade dos adultos entre $22^{\circ} \mathrm{C}$ e $28^{\circ} \mathrm{C}$. As $\mathrm{Tb}, \mathrm{K}$ de ovo a adulto, e o número de gerações anuais em campo foram de 11,$33 ; 8,99$ e $13,61^{\circ} \mathrm{C}, 192,3 ; 213,2$ e 116,5 graus-dias, e $23 ; 24,6$ e 30,3 gerações para as populações de A. aegypti de Boqueirão ( $07^{\circ} 29^{\prime} 27^{\prime \prime}$,

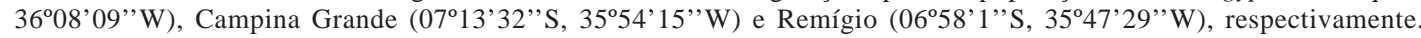

PALAVRAS-CHAVE. Insecta, Aedini, biologia, exigências térmicas.

Aedes (Stegomyia) aegypti (Linnaeus, 1762) é o mosquito mais importante na transmissão da febre amarela e dos quatro sorotipos do vírus da dengue (Lozover, 2001; Aldama \& Garcia, 2001; Forattini, 2002). É um inseto cosmopolita, bem adaptado ao ambiente urbano, distribuindo-se nas áreas tropicais e subtropicais, localizadas entre $45^{\circ}$ de latitude Norte e $40^{\circ}$ de latitude Sul (LoZover, 2001; ForatTinI, 2002).

Aedes aegypti desenvolveu em sua trajetória evolutiva um comportamento estritamente sinantrópico e antropofílico, sendo reconhecido entre os culicídeos como a espécie mais associada ao homem (NATAL, 2002). Múltiplos são os fatores envolvidos na dispersão de culicídeos domiciliados. A expansão geográfica das populações do A. aegypti sofre influência de fatores sociais e ambientais, entre os quais a densidade demográfica, a atividade econômica e o clima. A influência do clima na distribuição e abundância dos insetos e na epidemiologia das áreas de ocorrência de doenças transmitidas por vetores em vários continentes e seu agravamento tiveram, entre outros determinantes, o aumento da temperatura do planeta, especialmente nos últimos 100 anos (Glasser \& GoMes, 2002).
No estado de São Paulo foi verificada uma forte associação entre a temperatura média de julho e o estabelecimento das populações do A. aegypti, sendo que a velocidade de ocupação de novos municípios foi tanto maior quanto maior a faixa de temperatura. Em regiões com temperaturas acima de $16-18^{\circ} \mathrm{C}$, em menos de 2 anos após o estabelecimento do vetor $10 \%$ dos municípios estavam infestados, elevando-se para $90 \%$ após cinco anos, como por exemplo as mesorregiões de Araçatuba, Presidente Prudente, Ribeirão Preto e São José do Rio Preto onde as isotermas de julho foram acima de $18^{\circ} \mathrm{C}$ (Glasser \& Gomes, 2002). Em Nova Iguaçu (Rio de Janeiro), a frequência de $A$. aegypti foi maior nos meses de dezembro e fevereiro, os quais foram os meses mais quentes, com temperaturas médias acima de $25^{\circ}$ e de maior precipitação pluviométrica no verão, sendo o menor período de ocorrência os meses de inverno (HonORIO \& Lourenço-De-Oliveira, 2001). No estado da Paraíba a amplitude de temperatura favorável ao ciclo de vida das populações de A. aegypti encontra-se entre $22^{\circ} \mathrm{C}$ e $30^{\circ} \mathrm{C}$, podendo ocorrer sob essas condições acima de vinte gerações do inseto ao longo do ano (BESERRA et al., 2006). 
A temperatura é um importante fator ecológico que influencia o estabelecimento das populações de insetos, seja diretamente através do seu desenvolvimento, ou indiretamente através de sua alimentação (SILVEIRA-NETO et al., 1976). Com base em estudos de temperatura que determinem as exigências térmicas para o desenvolvimento de A. aegypti e de representações climáticas das diversas regiões de ocorrência do mosquito através de normais climatológicas é possível se ter um melhor entendimento da sua dinâmica populacional e prever o número de gerações anuais, bem como as épocas de maior ocorrência nas áreas de infestação. Dessa forma, é fundamental o desenvolvimento de estudos bioecológicos que auxiliem no entendimento da dinâmica populacional do mosquito, para que se possa desenvolver modelos preditivos de ocorrência de A. aegypti nas áreas vulneráveis a infestações. Esta pesquisa objetivou avaliar o efeito da temperatura sobre o ciclo de vida de A. aegypti, determinar as exigências térmicas para o seu desenvolvimento e estimar o número de gerações anuais do inseto em campo.

\section{MATERIAL E MÉTODOS}

As criações das populações de A. aegypti e os bioensaios de laboratório foram conduzidos no laboratório de Controle Biológico pertencente ao Núcleo de Manejo Integrado de Pragas da Universidade Estadual da Paraíba, utilizando-se a primeira geração de laboratório de amostras de três populações do vetor coletadas nos municípios de Boqueirão (07²9'27' S, 3608'09'W), Campina Grande (07 13 '32' S, 35 54 '15' 'W) e Remígio (06 58' ' 'S, 35'47'29''W) (Fig. 1). As amostras das populações do mosquito foram obtidas a partir de ovos coletados em armadilhas ovitrampas instaladas no intra e peridomicílio de residências escolhidas aleatoriamente em dez quarteirões, sendo instaladas 50 armadilhas no Bairro do Pedegral (Campina Grande) e 30 armadilhas no Bairro Bela vista em Boqueirão e no Centro de Remígio.

As palhetas de eucatex das armadilhas ovitrampas contendo ovos de campo de A. aegypti foram colocadas para secar por um período de 48 horas sendo em seguida acondicionadas em bandejas plásticas de cor branca medindo $40 \mathrm{~cm}$ de comprimento x $27 \mathrm{~cm}$ de largura x 7,5 $\mathrm{cm}$ de profundidade com um terço da sua capacidade preenchida com água desclorada. Após a eclosão, foi oferecida ração para peixe ornamental na proporção de
$100 \mathrm{mg} / \mathrm{bandeja}$, a cada três dias e as bandejas cobertas por uma tela de malha fina. As pupas foram sexadas segundo FORATTINI (2002) e posteriormente transferidas em copos descartáveis de $250 \mathrm{ml}$ para as gaiolas de criação dos adultos. Essas gaiolas, construídas de armação de madeira e tecido tipo organza medindo $40 \mathrm{~cm}$ x $40 \mathrm{~cm}$ x 20 cm de fundo, receberam 100 indivíduos, na proporção de um macho para cada fêmea. Aos adultos ofertou-se uma solução de mel a $20 \%$ e às fêmeas foi permitido repasto sanguíneo em codornas, Coturnix japonica (Temminck \& Schlegel, 1849) durante trinta minutos, três vezes por semana. Após o repasto colocou-se, em cada gaiola, um copo descartável de $250 \mathrm{ml}$ com água desclorada, com um funil plástico revestido por um papel filtro para servir como substrato de oviposição.

Ciclo de vida de $A$. aegypti em seis temperaturas constantes. O ciclo biológico de cada população foi estudado em câmaras climatizadas reguladas a $18 \pm 2^{\circ} \mathrm{C}$, $22 \pm 2{ }^{\circ} \mathrm{C}, 26 \pm 2{ }^{\circ} \mathrm{C}, 28 \pm 2{ }^{\circ} \mathrm{C}, 32 \pm 2{ }^{\circ} \mathrm{Ce} 34 \pm 2{ }^{\circ} \mathrm{C}$ e fotofase de 12 horas. Para o estudo do desenvolvimento larval foram colocados, para cada temperatura, 5 copos de polietileno de $250 \mathrm{ml}$, contendo 10 larvas $\left(\mathrm{F}_{1}\right)$ de $1^{\circ}$ estádio de cada população, repetidos cinco vezes. Para as larvas ofertou-se, diariamente, $10 \mathrm{mg}$ de ração para peixe. Duas vezes ao dia foi aferida a temperatura da água dos copos, usando-se um termômetro de mercúrio com variação de $0^{\circ} \mathrm{C}$ a $60^{\circ} \mathrm{C}$. Os adultos foram mantidos em gaiolas de madeira telada $\left(20 \mathrm{~cm}^{3}\right)$ contendo vinte casais, em cinco repetições, sendo oferecida uma solução de mel a $20 \%$, além de permitir às fêmeas o repasto sanguíneo em codornas durante 20 minutos, três vezes por semana. Para a fase de ovo foram avaliadas as 20 primeiras posturas de cada tratamento. Estas posturas foram acondicionadas em Placa de Petri de vidro ( $15 \mathrm{~cm}$ de diâmetro x $2,5 \mathrm{~cm}$ de profundidade) com água desclorada, considerando-se como inviáveis os ovos que até o $20^{\circ}$ dia não tiveram larvas eclodidas. Foram avaliados o período de desenvolvimento e a viabilidades das fases de ovo, larva e pupa, a razão sexual e a longevidade e fecundidade da fase adulta.

O delineamento experimental foi inteiramente casualizado, sendo os dados analisados em esquema fatorial $3 \times 6$, três populações e seis temperaturas, para os estágios de ovo, larva, pupa e adulto de A. aegypti. As variáveis biológicas avaliadas foram submetidas à análise de variância e as médias comparadas pelo teste de Tukey $(\mathrm{p}<0,05)$

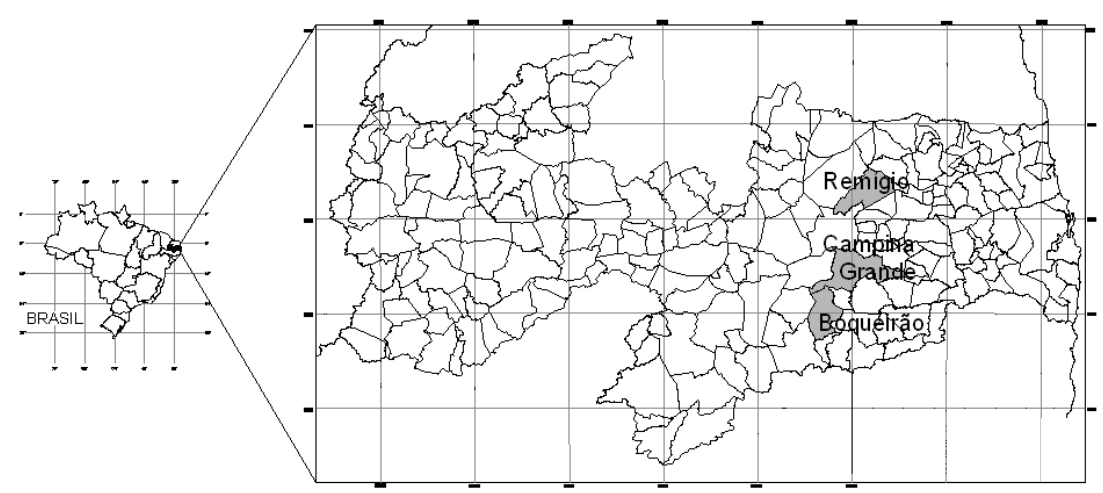

Figura 1. Mapa do Estado da Paraíba indicando os locais de coleta de Aedes aegypti (Linnaeus, 1762). 
Necessidades térmicas para o desenvolvimento e estimativa do número de gerações anuais de $A$. aegypti. Com base na duração do desenvolvimento dos imaturos e do ciclo total obtido a $18 \pm 2^{\circ} \mathrm{C}, 22 \pm 2^{\circ} \mathrm{C}, 26 \pm 2^{\circ} \mathrm{C}, 28 \pm$ $2{ }^{\circ} \mathrm{C}, 32 \pm 2{ }^{\circ} \mathrm{C}$ e $34 \pm 2{ }^{\circ} \mathrm{C}$ foram determinados os limites térmicos inferiores de desenvolvimento (tb) e as constantes térmicas $(\mathrm{K})$ através do método da hipérbole (HADDAD \& PARRA, 1984) dado pela equação $\mathrm{K}=\mathrm{y}(\mathrm{t}-\mathrm{tb})$ onde: $\mathrm{K}$ é a constante térmica, y o tempo de desenvolvimento (dias), t a temperatura ambiente $\left({ }^{\circ} \mathrm{C}\right)$, e tb a temperatura do limiar de desenvolvimento. A partir dos dados biológicos obtidos na temperatura na qual o ciclo foi completado em menor tempo e em função da constante térmica do ciclo biológico, do total de grausdias disponíveis para o desenvolvimento e nas normais térmicas das áreas de estudo, foi estimado o número de gerações anuais do inseto, em laboratório e no campo. $\mathrm{O}$ número de gerações anuais do inseto foi estimado com base em suas exigências térmicas, através da equação $\mathrm{NG}=\mathrm{T}(\mathrm{tc}-\mathrm{tb}) / \mathrm{K}$, onde $\mathrm{T}$ é o tempo considerado em anos e tc as temperaturas médias mensais das regiões de estudo, sendo $\mathrm{K}$ e tb os parâmetros já definidos anteriormente.

\section{RESULTADOS}

Ciclo de vida de $A$. aegypti em seis temperaturas constantes. As temperaturas médias das águas em que ocorreram os desenvolvimentos da fase de larva das três populações de $A$. aegypti estudadas variaram de $17,5^{\circ} \mathrm{C}$ a $17,7^{\circ} \mathrm{C} ; 21^{\circ} \mathrm{C}$ a $21,4^{\circ} \mathrm{C} ; 25^{\circ} \mathrm{C}$ a $25,5^{\circ} \mathrm{C} ; 27^{\circ} \mathrm{C}$ a $27,8^{\circ} \mathrm{C}$; $31^{\circ} \mathrm{C}$ a $31,5^{\circ} \mathrm{C}$ e de $32,5^{\circ} \mathrm{C}$ a $33^{\circ} \mathrm{C}$ para as temperaturas ambientes de $18^{\circ} \mathrm{C}, 22^{\circ} \mathrm{C}, 26^{\circ} \mathrm{C}, 28^{\circ} \mathrm{C}, 32^{\circ} \mathrm{C}$ e $34^{\circ} \mathrm{C}$ respectivamente.

A duração e viabilidade das fases de ovo, larva, pupa, de ovo à emergência do adulto, a longevidade dos adultos e a fecundidade das fêmeas foram dependentes do efeito conjunto da população e temperatura, revelando serem estas populações distintas quanto aos seus padrões de desenvolvimento e reprodução com relação à temperatura (Tabs. I-IV).

Os períodos de desenvolvimento de ovo, larva, pupa e de ovo a adulto diminuíram com a elevação térmica, sendo registrados nos extremos de temperaturas $\left(18\right.$ e $\left.34^{\circ} \mathrm{C}\right)$ os maiores e menores períodos de desenvolvimentos, respectivamente (Tabs. I e III). O período de incubação dos ovos das populações de $A$. aegypti variaram de 13,66 dias a $18^{\circ} \mathrm{C}$ a 2,93 dias a $34^{\circ} \mathrm{C}$. Porém deve-se ressaltar que, embora tenha ocorrido interação significativa entre população e temperatura, em geral não houveram diferenças significativas com relação ao período de desenvolvimento embrionário, sendo este de 6,19; 5,73 e 6,44 dias para as populações de Boqueirão, Campina Grande e Remígio, respectivamente. Para essas populações só ocorreram diferenças significativas quanto ao período de desenvolvimento embrionário nas temperaturas de $32^{\circ}$ e $34^{\circ} \mathrm{C}$ (Tab. I).

Embora não se tenha detectado diferenças significativas com relação ao período de desenvolvimento embrionário das populações de Boqueirão, Campina Grande e Remígio, constatou-se que o tempo de desenvolvimento para as fases de larva, pupa e ovo a adulto diferiu significativamente entre estas em todas as temperaturas avaliadas, sendo os menores períodos observados para a população de Campina Grande (com média geral de 4,69; 1,87 e 9,27 para as fases de larva, pupa e ovo a adulto, respectivamente) (Tab. III)

A viabilidade da fase ovo de $A$. aegypti da população de Remígio foi a mais afetada pelas temperaturas extremas de $18^{\circ} \mathrm{Ce} 34^{\circ} \mathrm{C}$, ocorrendo em média $45,4 \%$ e $43,1 \%$ de sobrevivência respectivamente. Para a população de Campina Grande a sobrevivência foi acima de $70 \%$, com média geral de 78,7\% (Tab. II). Embora a temperatura tenha afetado o desenvolvimento embrionário de A. aegypti, teve pouco efeito sobre as viabilidades das fases de larva e pupa e, com exceção da viabilidade de $57,6 \%$ observada para a fase de pupa da população de Campina Grande a $28^{\circ} \mathrm{C}$, em todas as demais temperaturas a sobrevivência foi alta, acima de $80 \%$, com média geral $90,7 \% ; 99,8 \%$ e $99,4 \%$ para as fases de larva e de $95,1 \% ; 85,9 \%$ e $95,9 \%$ para a fase de pupa das populações de Boqueirão, Campina Grande e Remígio, respectivamente (Tab. II).

Constataram-se interações significativas entre população e temperatura com relação a longevidade e fecundidade dos adultos, havendo uma diminuição das longevidades com o aumento da temperatura a partir dos $28^{\circ} \mathrm{C}$, sendo significativamente maiores a $26^{\circ} \mathrm{C}$ para as fêmeas e a $22^{\circ}$ e $26^{\circ} \mathrm{C}$ para os machos das três populações. Considerando-se a média geral das longevidades em todos os tratamentos não se detectou diferenças significativas entre as populações, sendo esta de 21,2; 21,1 e 22,6 para as fêmeas e de 20,1; 22,3 e 21,7 para os machos das populações de Boqueirão, Campina Grande e Remígio, respectivamente (Tab. IV). Os extremos de temperatura de $18^{\circ} \mathrm{Ce} 34^{\circ} \mathrm{C}$ diminuíram consideravelmente o número de ovos por fêmea, principalmente para a população de Campina Grande. Nesta localidade obtevese o menor número de ovos/fêmeas, cuja média a $18^{\circ} \mathrm{C}$ foi de 3,9 ovos e a $34^{\circ} \mathrm{C}$ de 3,4 ovos, com média geral de 52

Tabela I. Duração (dias) (X \pm EP) do desenvolvimento embrionário de populações de Aedes aegypti (Linnaeus, 1762) dos municípios de Boqueirão, Campina Grande e Remígio na Paraíba, em seis temperaturas e fotofase de 12 horas ( ${ }^{1}$, médias originais; para efeito da análise estatística os dados foram transformados em $\sqrt{x+0,5} ;{ }^{2}$, médias seguidas da mesma letra minúscula nas linhas e maiúsculas nas colunas, não diferem entre si pelo teste de Tukey $(\mathrm{p}<0,05))$.

\begin{tabular}{|c|c|c|c|c|c|c|c|}
\hline \multirow[t]{2}{*}{ População } & \multicolumn{7}{|c|}{ Desenvolvimento embrionário (dias)/Temperatura ${ }^{1 ; 2}$} \\
\hline & $18^{\circ} \mathrm{C}$ & $22^{\circ} \mathrm{C}$ & $26^{\circ} \mathrm{C}$ & $28^{\circ} \mathrm{C}$ & $32^{\circ} \mathrm{C}$ & $34^{\circ} \mathrm{C}$ & Média Geral \\
\hline Boqueirão & $13,66 \pm 0,41 \mathrm{aA}$ & $6,99 \pm 0,54 \mathrm{bA}$ & $6,12 \pm 0,25 \mathrm{bA}$ & $3,17 \pm 0,27 \mathrm{dA}$ & $2,94 \pm 0,17 \mathrm{~dB}$ & $4,24 \pm 0,32 \mathrm{cdB}$ & $6,19 \pm 0,13 \mathrm{~A}$ \\
\hline Campina Grande & $11,32 \mathrm{aA}$ & $8,82 \pm 0,64 \mathrm{aA}$ & $4,77 \pm 0,20 \mathrm{bA}$ & $3,11 \pm 0,19 \mathrm{bA}$ & $3,43 \pm 0,43 \mathrm{bB}$ & $2,93 \pm 0,77 \mathrm{bB}$ & $5,73 \pm 0,19 \mathrm{~A}$ \\
\hline Remígio & $11,15 \pm 0,70 \mathrm{aA}$ & $8,45 \pm 0,64 \mathrm{aA}$ & $5,52 \pm 0,52 \mathrm{cA}$ & $3,43 \pm 0,21 \mathrm{cA}$ & $4,51 \pm 0,39 \mathrm{cA}$ & $5,63 \pm 0,84 \mathrm{bcA}$ & $6,44 \pm 0,35 \mathrm{~A}$ \\
\hline
\end{tabular}

$\mathrm{CV}(\%)=14,20$ 
Tabela II. Viabilidade (\%) das fases de ovo, larva e pupa de populações de Aedes aegypti (Linnaeus, 1762) dos municípios de Boqueirão, Campina Grande e Remígio na Paraíba em seis temperaturas e fotofase de 12 horas ( ${ }^{1}$, médias seguidas da mesma letra minúscula nas linhas e maiúsculas nas colunas não diferem entre si pelo teste de Tukey ( $\mathrm{p}<0,05)$; ${ }^{2}$, variação da temperatura da água em que ocorreu $o$ desenvolvimento larval de Aedes aegypti; ${ }^{3}$, temperatura ambiente).

\begin{tabular}{|c|c|c|c|c|c|c|c|}
\hline População & $18^{\circ} \mathrm{C}$ & $22^{\circ} \mathrm{C}$ & $26^{\circ} \mathrm{C}$ & $28^{\circ} \mathrm{C}$ & $32^{\circ} \mathrm{C}$ & $34^{\circ} \mathrm{C}$ & Média Geral \\
\hline \multicolumn{8}{|c|}{ 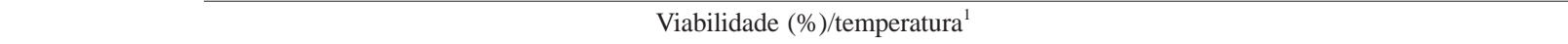 } \\
\hline \multicolumn{8}{|c|}{ Fase ovo } \\
\hline Boqueirão & $42,07 \pm 7,9 \mathrm{cB}$ & $53,75 \pm 7,5 \mathrm{bcA}$ & $89,98 \pm 3,1 \mathrm{abA}$ & $100 \mathrm{aA}$ & $97,35 \pm 0,97 \mathrm{aA}$ & $70,01 \pm 4,8 \mathrm{abcA}$ & $75,52 \pm 2,02 \mathrm{~A}$ \\
\hline Campina Grande & $76,76 \pm \mathrm{aA}$ & $79,42 \pm 5 \mathrm{aA}$ & $93,71 \pm 4,14 \mathrm{aA}$ & $87,82 \pm 5,9 \mathrm{aA}$ & $64,81 \pm 10,7 \mathrm{aB}$ & $70,0 \pm 23,80 \mathrm{aA}$ & $78,75 \pm 2,99 \mathrm{~A}$ \\
\hline Remígio & $45,46 \pm 8,56 \mathrm{bB}$ & $69,66 \pm 5,8 \mathrm{abA}$ & $84,26 \pm 4,42 \mathrm{aA}$ & $90,39 \pm 4,89 \mathrm{aA}$ & $69,73 \pm 6 \mathrm{abAB}$ & $43,12 \pm 14,6 \mathrm{bB}$ & $67,10 \pm 2,61 \mathrm{~A}$ \\
\hline \multicolumn{8}{|l|}{$\mathrm{CV}=33,66 \%$} \\
\hline \multicolumn{8}{|c|}{ Fase larva } \\
\hline & $\left(17,5-17,7^{\circ} \mathrm{C}\right)^{2}$ & $\left(21,0-21,4^{\circ} \mathrm{C}\right)$ & $\left(25,0-25,5^{\circ} \mathrm{C}\right)$ & $\left(27,0-27,8^{\circ} \mathrm{C}\right)$ & $\left(31,0-31,5^{\circ} \mathrm{C}\right)$ & $\left(32,5-33,0^{\circ} \mathrm{C}\right)^{2}$ & \\
\hline Boqueirão & $98,33 \pm 1,3 \mathrm{abA}$ & $99,33 \pm 0,42 \mathrm{aA}$ & $96,16 \pm 0,6 \mathrm{abA}$ & $91,33 \pm 2,6 \mathrm{bcB}$ & $72,00 \pm 6,3 \mathrm{~dB}$ & $87,53 \pm 2,1 \mathrm{cB}$ & $90,75 \pm 1,95 \mathrm{~B}$ \\
\hline Campina Grande & $100 \mathrm{aA}$ & $99,66 \pm 0,33 \mathrm{aA}$ & $99,66 \pm 0,3 \mathrm{aA}$ & $100 \mathrm{aA}$ & $100 \mathrm{aA}$ & $100 \mathrm{aA}$ & $99,88 \pm 0,77 \mathrm{~A}$ \\
\hline Remígio & $100 \mathrm{aA}$ & $99,66 \pm 0,33 \mathrm{aA}$ & $98,33 \pm 0,8 \mathrm{aA}$ & $100 \mathrm{aA}$ & $99,00 \pm 0,44 \mathrm{aA}$ & $99,66 \pm 0,33 \mathrm{aA}$ & $99,44 \pm 4,39 \mathrm{~A}$ \\
\hline \multicolumn{8}{|l|}{$\mathrm{CV}=4,44 \%$} \\
\hline \multicolumn{8}{|c|}{ Fase de pupa } \\
\hline Boqueirão & $92,12 \pm 3,2 \mathrm{aA}$ & $94,86 \pm 1,5 \mathrm{aAB}$ & $96,23 \pm 1,6 \mathrm{aA}$ & $99,62 \pm 1,8 \mathrm{aA}$ & $92,88 \pm 2,3 \mathrm{aA}$ & $92,88 \pm 2,3 \mathrm{aA}$ & $95,19 \pm 0,8 \mathrm{~A}$ \\
\hline Campina Grande & $94,00 \pm 1,2 \mathrm{aA}$ & $88,66 \pm 1,9 \mathrm{aB}$ & $94,00 \pm 1,2 \mathrm{aA}$ & $57,66 \pm 0,9 \mathrm{bB}$ & $92,16 \pm 1,7 \mathrm{aA}$ & $89,00 \pm 3,4 \mathrm{aA}$ & $85,91 \pm 2,3 \mathrm{~B}$ \\
\hline Remígio & $93,33 \pm 1,6 \mathrm{aA}$ & $96,33 \pm 0,6 \mathrm{aA}$ & $97,61 \pm 0,9 \mathrm{aA}$ & $99,00 \pm 0,6 \mathrm{aA}$ & $97,33 \pm 0,8 \mathrm{aA}$ & $92,33 \pm 1,6 \mathrm{aA}$ & $95,99 \pm 0,5 \mathrm{~A}$ \\
\hline
\end{tabular}

Tabela III. Duração (dias) das fases de larva, pupa e de ovo à emergência do adulto de populações de Aedes aegypti (Linnaeus, 1762) dos municípios de Boqueirão, Campina Grande e Remígio na Paraíba em seis temperaturas e fotofase de 12 horas $\left({ }^{1}\right.$, médias seguidas da mesma letra minúscula nas linhas e maiúsculas nas colunas, não diferem entre si pelo teste de Tukey $(\mathrm{p}<0,05) ;{ }^{2}$, variação da temperatura da água em que ocorreu o desenvolvimento larval de Aedes aegypti).

\begin{tabular}{|c|c|c|c|c|c|c|c|}
\hline \multicolumn{8}{|c|}{ Fases de desenvolvimento/temperatura ${ }^{1}$} \\
\hline \multirow[t]{2}{*}{ População } & $18^{\circ} \mathrm{C}$ & $22^{\circ} \mathrm{C}$ & $26^{\circ} \mathrm{C}$ & $28^{\circ} \mathrm{C}$ & $32^{\circ} \mathrm{C}$ & $34^{\circ} \mathrm{C}$ & Média Geral \\
\hline & $\left(17,5-17,7^{\circ} \mathrm{C}\right)^{2}$ & $\left(21,0-21,4^{\circ} \mathrm{C}\right)$ & $\left(25,0-25,5^{\circ} \mathrm{C}\right)$ & $\left(27,0-27,8^{\circ} \mathrm{C}\right)$ & $\left(31,0-31,5^{\circ} \mathrm{C}\right)$ & $\left(32,5-33,0^{\circ} \mathrm{C}\right)$ & \\
\hline \multicolumn{8}{|c|}{ Fase larval } \\
\hline Boqueirão & $12,69 \pm 0,15 \mathrm{aB}$ & $7,53 \pm 0,24 \mathrm{bA}$ & $5,69 \pm 0,29 \mathrm{cA}$ & $5,43 \pm 0,05 \mathrm{cA}$ & $4,67 \pm 0,23 \mathrm{dA}$ & $3,68 \pm 0,06 \mathrm{cA}$ & $6,62 \pm 0,5 \mathrm{~A}$ \\
\hline Campina Grande & $7,41 \pm 0,08 \mathrm{aC}$ & $6,14 \pm 0,01 \mathrm{bB}$ & $4,48 \pm 0,05 \mathrm{cB}$ & $4,14 \pm 0,15 \mathrm{cB}$ & $3,01 \pm 0,20 \mathrm{dC}$ & $3,00 \pm 0,01 \mathrm{~dB}$ & $4,69 \pm 0,24 \mathrm{C}$ \\
\hline Remígio & $14,12 \pm 1,4 \mathrm{aA}$ & $7,65 \pm 0,10 \mathrm{bA}$ & $5,27 \pm 0,04 \mathrm{cA}$ & $3,93 \pm 0,04 \mathrm{~dB}$ & $3,59 \pm 0,03 \mathrm{~dB}$ & $3,52 \pm 0,09 \mathrm{dA}$ & $6,35 \pm 0,70 \mathrm{~B}$ \\
\hline \multicolumn{8}{|l|}{$\mathrm{CV}=6,03 \%$} \\
\hline \multicolumn{8}{|c|}{ Fase de pupa } \\
\hline Boqueirão & $5,86 \pm 0,06 \mathrm{aA}$ & $3,60 \pm 0,04 \mathrm{bA}$ & $2,06 \pm 0,02 \mathrm{cA}$ & $2,0 \pm 0,004 \mathrm{cA}$ & $1,72 \pm 0,04 \mathrm{cdA}$ & $1,49 \pm 0,01 \mathrm{dA}$ & $2,79 \pm 0,25 \mathrm{~A}$ \\
\hline Campina Grande & $3,46 \pm 0,14 \mathrm{aB}$ & $2,79 \pm 0,27 \mathrm{bB}$ & $1,16 \pm 0,10 \mathrm{cB}$ & $1,2 \pm 0,11 \mathrm{cB}$ & $1,11 \pm 0,02 \mathrm{cB}$ & $1,50 \pm 0,09 \mathrm{cA}$ & $1,87 \pm 0,16 \mathrm{C}$ \\
\hline Remígio & $5,61 \pm 0,25 \mathrm{aA}$ & $3,30 \pm 0,03 \mathrm{bA}$ & $1,91 \pm 0,07 \mathrm{cA}$ & $1,8 \pm 0,03 \mathrm{cdA}$ & $1,60 \pm 0,04 \mathrm{cdB}$ & $1,35 \pm 0,06 \mathrm{dA}$ & $2,59 \pm 0,25 \mathrm{~B}$ \\
\hline \multicolumn{8}{|l|}{$\mathrm{CV}=11,21 \%$} \\
\hline \multicolumn{8}{|c|}{ Ovo-adulto } \\
\hline Boqueirão & $33,38 \pm 1,79 \mathrm{aA}$ & $18,39 \pm 0,91 \mathrm{bA}$ & $14,08 \pm 0,6 \mathrm{bcA}$ & $10,19 \pm 0,14 \mathrm{cA}$ & $9,39 \pm 0,40 \mathrm{cA}$ & $9,53 \pm 0,59 \mathrm{cAB}$ & $15,82 \pm 1,30 \mathrm{~A}$ \\
\hline Campina Grande & $7,49 \pm 0,002 \mathrm{bB}$ & $17,55 \pm 0,68 \mathrm{aA}$ & $10,38 \pm 0,38 \mathrm{bA}$ & $7,38 \pm 0,38 \mathrm{bA}$ & $6,50 \pm 0,36 \mathrm{bA}$ & $6,33 \pm 1,10 \mathrm{bB}$ & $9,27 \pm 0,95 \mathrm{~B}$ \\
\hline Remígio & $29,95 \pm 0,79 \mathrm{aA}$ & $18,81 \pm 0,70 \mathrm{bA}$ & $12,15 \pm 0,60 \mathrm{cA}$ & $8,98 \pm 0,20 \mathrm{cA}$ & $9,18 \pm 0,41 \mathrm{cA}$ & $10,58 \pm 0,87 \mathrm{cA}$ & $16,94 \pm 1,13 \mathrm{~A}$ \\
\hline
\end{tabular}

$\mathrm{CV}=19,35 \%$

ovos/fêmeas. O maior número de ovos/fêmea foi constatado a $26^{\circ} \mathrm{C}$ para as fêmeas da população de Boqueirão, com média de 610,6 ovos. Considerando-se a média geral de ovos/fêmeas de todas as populações, as fêmeas da população de Boqueirão, com 156,7 ovos/ fêmea, foram mais fecundas (Tab. IV).

Necessidades térmicas para o desenvolvimento e estimativa do número de gerações anuais de A. aegypti. Verificou-se que a velocidade de desenvolvimento em função da temperatura ajustou-se ao modelo linear determinado através da recíproca da equação da hipérbole (HADDAD \& PARRA, 1984). Os limites térmicos inferiores de desenvolvimento (tb) para as fases de ovo, larva, pupa e de ovo à emergência do adulto foram de 9,$21 ; 10,05 ; 12,12^{\circ} \mathrm{C}$ e 11,$33 ; 3,54 ; 11,84 ; 12,38$ e $8,99^{\circ} \mathrm{Ce}$ 14,$74 ; 9,09 ; 6,73$ e $13,61^{\circ} \mathrm{C}$ para as populações de $A$. aegypti de Boqueirão, Campina Grande e Remígio, respectivamente. Dentre as fases de desenvolvimento do vetor, as fases de ovo e de ovo à emergência do adulto da população de Campina Grande e de larva e pupa da população de Remígio, demonstraram maiores tolerâncias à baixa temperatura, pois apresentaram as menores temperaturas bases. Essas fases também apresentaram maiores velocidades de desenvolvimento se comparadas às fases de desenvolvimento da população de Boqueirão, o que é evidenciado pelos maiores coeficientes angulares 
estimados pelas equações de regressão (Tab. V). As constantes térmicas estimadas para as fases de ovo, larva, pupa e de ovo à emergência do adulto foram de 79,61; 89,$76 ; 32,53$ e 192,30 graus-dia (GD), 117,65; 69,20; 29, 15 e 213,20 (GD) e 46,19; 71,89; 29,85 e 116,55 GD para as populações de A. aegypti de Boqueirão, Campina Grande e Remígio, respectivamente. A constante térmica representa a somatória das temperaturas favoráveis ao desenvolvimento dos insetos durante esse período, ou seja, as temperaturas que estão acima da temperatura base. Neste estudo foram requeridos 192,3; 213,2 e 116,5 GD para completar os desenvolvimentos dos ciclos aquáticos de A. aegypti provenientes de Boqueirão, Campina Grande e Remígio, respectivamente.
Tomando-se por base as exigências térmicas para o desenvolvimento e as normais térmicas de cada região, o número de gerações anuais em campo é de $23 ; 24,6$ e 30,3 , sendo que em condições de laboratório, a $26^{\circ} \mathrm{C}$, esse número é de 27,$8 ; 29,1$ e 38,8 para as populações de Boqueirão, Campina Grande e Remígio, respectivamente. Por conseguinte, verificou-se que no município de Remígio A. aegypti apresenta um maior número de gerações anuais do que nas demais localidades. Tal fato está associado à maior velocidade de desenvolvimento da população de Remígio, como pode ser verificado pelo maior coeficiente angular das equações de regressão estimadas (Tab. V) possibilitando um maior número de gerações ao longo do ano.

Tabela IV. Longevidade dos adultos (dias) e número de ovos/fêmea de populações de Aedes aegypti (Linnaeus, 1762) dos municípios de Boqueirão, Campina Grande e Remígio na Paraíba em seis temperaturas e fotofase de 12 horas ( ${ }^{1}$, médias seguidas da mesma letra minúscula nas linhas e maiúsculas nas colunas não diferem entre si pelo teste de Tukey $(\mathrm{p}<0,05)$; ${ }^{2,3}$, médias originais; para efeito da análise estatística os dados foram transformados em $\log (\mathrm{x}+1))$.

\begin{tabular}{|c|c|c|c|c|c|c|c|}
\hline \multirow[b]{2}{*}{ População } & \multicolumn{6}{|c|}{ Temperatura $^{1,2}$} & \multirow[b]{2}{*}{ Média Geral } \\
\hline & $18^{\circ} \mathrm{C}$ & $22^{\circ} \mathrm{C}$ & $26^{\circ} \mathrm{C}$ & $28^{\circ} \mathrm{C}$ & $32^{\circ} \mathrm{C}$ & $34^{\circ} \mathrm{C}$ & \\
\hline \multicolumn{8}{|c|}{ Longevidade dos machos } \\
\hline Boqueirão & $22,50 \pm 1,9 \mathrm{cAB}$ & $29,24 \pm 1,5 \mathrm{abAB}$ & $33,31 \pm 1,3 \mathrm{aB}$ & $14,22 \pm 07 \mathrm{cdA}$ & $10,16 \pm 1,3 \mathrm{dA}$ & $17,94 \pm 3,3 \mathrm{cdA}$ & $21,22 \pm 0,76 \mathrm{~A}$ \\
\hline Campina Grande & $6,40 \pm 1,6 \mathrm{bcB}$ & $33,22 \pm 1,9 \mathrm{aA}$ & $33,56 \pm 2,3 \mathrm{aB}$ & $19,94 \pm 1,4 \mathrm{bA}$ & $13,28 \pm 3,0 \mathrm{bcA}$ & $10,30 \pm 0,9 \mathrm{cB}$ & $21,11 \pm 1,90 \mathrm{~A}$ \\
\hline Remígio & $25,78 \pm 2,1 \mathrm{bA}$ & $23,70 \pm 3,4 \mathrm{bB}$ & $47,08 \pm 1,9 \mathrm{aA}$ & $20,64 \pm 1,3 \mathrm{bA}$ & $10,06 \pm 0,7 \mathrm{cA}$ & $8,84 \pm 0,6 \mathrm{cB}$ & $22,68 \pm 2,20 \mathrm{~A}$ \\
\hline \multicolumn{8}{|l|}{$\mathrm{CV}=11,94 \%$} \\
\hline \multicolumn{8}{|c|}{ Longevidade de fêmeas } \\
\hline Boqueirão & $21,90 \pm 1,6 \mathrm{abA}$ & $27,52 \pm 2,4 \mathrm{aA}$ & $25,46 \pm 4,2 \mathrm{aB}$ & $13,81 \pm 0,5 \mathrm{bB}$ & $13,44 \pm 0,6 \mathrm{bA}$ & $13,48 \pm 1,2 \mathrm{abA}$ & $20,10 \pm 1,1 \mathrm{~A}$ \\
\hline Campina Grande & $15,71 \pm 2,5 \mathrm{cA}$ & $27,30 \pm 1,9 \mathrm{abA}$ & $40,54 \pm 2,1 \mathrm{aA}$ & $21,44 \pm 1,5 \mathrm{bcAB}$ & $16,76 \pm 3,6 \mathrm{cA}$ & $12,16 \pm 1,4 \mathrm{cA}$ & $22,31 \pm 2,0 \mathrm{~A}$ \\
\hline Remígio & $22,67 \pm 0,3 \mathrm{bA}$ & $24,74 \pm 1,7 \mathrm{abA}$ & $38,36 \pm 2,7 \mathrm{aA}$ & $22,36 \pm 2,3 \mathrm{bA}$ & $11,22 \pm 1,1 \mathrm{cA}$ & $10,92 \pm 0,7 \mathrm{cA}$ & $21,71 \pm 1,7 \mathrm{~A}$ \\
\hline \multicolumn{8}{|l|}{$\mathrm{CV}=4,46 \%$} \\
\hline \multicolumn{8}{|c|}{ Número de ovos/fêmea ${ }^{3}$} \\
\hline Boqueirão & $46,9 \pm 16,2 \mathrm{bA}$ & $67,4 \pm 19,65 \mathrm{bA} \quad 6$ & $610,6 \pm 121,6 \mathrm{bA}$ & $66,6 \pm 23,15 \mathrm{bB}$ & $50,6 \pm 10,5 \mathrm{bA}$ & $97,9 \pm 16,1 \mathrm{bA}$ & $156,7 \pm 23,35 \mathrm{~A}$ \\
\hline Campina Grande & $3,9 \mathrm{bB}$ & $117,9 \pm 37,6 \mathrm{aA} \quad 8$ & $89,4 \pm 24,7 \mathrm{aB}$ & $84,1 \pm 29,8 \mathrm{aAB}$ & $13,4 \pm 8,7 \mathrm{bB}$ & $3,4 \pm 2,8 \mathrm{bB}$ & $52,0 \pm 9,99 \mathrm{~B}$ \\
\hline Remígio & $69,7 \pm 28,4 \mathrm{bcA}$ & $14,8 \pm 7,1 \mathrm{cdA} \quad 2$ & $214,7 \pm 27,9 \mathrm{abAB}$ & $389,2 \pm 150,8 \mathrm{aA}$ & $25,3 \pm 11,1 \mathrm{cdAB}$ & $1,8 \mathrm{~dB}$ & $119,2 \pm 28,58 \mathrm{~B}$ \\
\hline
\end{tabular}

Tabela V. Temperatura base (tb), constante térmica (K), intercepto (a) e coeficiente angular (b) da equação de regressão da velocidade de desenvolvimento e coeficiente de determinação $\left(\mathrm{R}^{2}\right)$ do desenvolvimento de populações de Aedes aegypti (Linnaeus, 1762) dos municípios de Boqueirão, Campina Grande e Remígio na Paraíba e fotofase de 12 horas.

\begin{tabular}{|c|c|c|c|c|c|}
\hline \multirow[t]{2}{*}{ População } & \multicolumn{5}{|c|}{ Fases de Desenvolvimento } \\
\hline & tb $\left({ }^{\circ} \mathrm{C}\right)$ & $\mathrm{K}(\mathrm{GD})$ & $\mathrm{a} \pm$ erro padrão & $\mathrm{b} \pm$ erro padrão & $\mathrm{R}^{2}$ \\
\hline & \multicolumn{5}{|c|}{ Fase de Ovo } \\
\hline Boqueirão & 9,21 & 79,61 & $-0,11570 \pm 0,13409$ & $0,01256 \pm 0,00492$ & 0,62 \\
\hline Campina Grande & 3,54 & 117,65 & $-0,03006 \pm 0,13077$ & $0,00850 \pm 0,00480$ & 0,44 \\
\hline \multirow[t]{2}{*}{ Remígio } & 14,74 & 46,19 & $-0,31917 \pm 0,07785$ & $0,02165 \pm 0,00260$ & 0,93 \\
\hline & \multicolumn{5}{|c|}{ Fase de Larva } \\
\hline Boqueirão & 10,05 & 89,76 & $-0,11205 \pm 0,03488$ & $0,01114 \pm 0,00132$ & 0,95 \\
\hline Campina Grande & 11,84 & 69,20 & $-0,17109 \pm 0,04741$ & $0,01445 \pm 0,00180$ & 0,94 \\
\hline \multirow[t]{2}{*}{ Remígio } & 9,09 & 71,89 & $-0,12641 \pm 0,04071$ & $0,01391 \pm 0,00153$ & 0,95 \\
\hline & \multicolumn{5}{|c|}{ Fase de pupa } \\
\hline Boqueirão & 12,12 & 32,53 & $-0,37208 \pm 0,07292$ & $0,03074 \pm 0,00268$ & 0,97 \\
\hline Campina Grande & 12,38 & 29,15 & $-0,42477 \pm 0,08704$ & $0,03430 \pm 0,00320$ & 0,97 \\
\hline \multirow[t]{2}{*}{ Remígio } & 6,73 & 29,85 & $-0,22559 \pm 0,41389$ & $0,03350 \pm 0,01520$ & 0,55 \\
\hline & \multicolumn{5}{|c|}{ Ovo-adulto } \\
\hline Boqueirão & 11,33 & 192,30 & $-0,05893 \pm 0,01574$ & $0,00520 \pm 0,000584$ & 0,95 \\
\hline Campina Grande & 8,99 & 213,20 & $-0,04226 \pm 0,03406$ & $0,00469 \pm 0,00127$ & 0,77 \\
\hline Remígio & 13,61 & 116,55 & $-0,11679 \pm 0,02243$ & $0,00858 \pm 0,000831$ & 0,96 \\
\hline
\end{tabular}




\section{DISCUSSÃO}

Os resultados mostraram que as populações de $A$. aegypti provenientes dos municípios de Boqueirão, Campina Grande e Remígio apresentaram padrões de desenvolvimento distintos em relação à temperatura e que o desenvolvimento das fases imaturas, a longevidade dos adultos e a fecundidade das fêmeas foram influenciados pelas temperaturas de 18, 22, 26, 28, 32 e 34 $\pm 2{ }^{\circ} \mathrm{C}$, coincidindo com os resultados de CALADO \& NAVARRo-SiLVA (2002a) para Aedes (S.) albopictus Skuse, 1894. Aqueles autores verificaram uma relação inversa entre tempo de desenvolvimento e temperatura onde os menores períodos de duração das fases de ovo, larva e pupa ocorreram com o aumento da temperatura. Para o período de incubação dos ovos houve uma redução de aproximadamente 27 dias quando os ovos foram mantidos a $25^{\circ} \mathrm{C}$ e $30^{\circ} \mathrm{C}$ em relação à temperatura de $15^{\circ} \mathrm{C}$. Essas diferenças, quanto ao padrão de desenvolvimento também foram detectadas para outras populações de $A$. aegypti provenientes dos municípios de Boqueirão, Brejo dos Santos, Campina Grande, Itaporanga e Remígio, todos na Paraíba, onde as diferenças encontradas quanto ao padrão de desenvolvimento foram consideradas inerentes a cada população, surgidas como consequiência das adaptações ecológicas às suas regiões de origem (BESERRA et al., 2006).

No presente estudo todas as temperaturas permitiram o desenvolvimento das populações de $A$. aegypti e não foram constatados efeitos negativos sobre as viabilidades das fases de ovo, larva e pupa (embora tenham influenciado o desenvolvimento das fases imaturas). Com exceção das viabilidades de ovo das populações de Boqueirão e Remígio a $18^{\circ}, 22^{\circ}$ e $34^{\circ} \mathrm{C}$ e da fase de pupa da população de Campina Grande a $28^{\circ} \mathrm{C}$, em todos os demais tratamentos observou-se mais de $70 \%$ de sobrevivência, superando assim os $90 \%$ para toda a fase larval de A. aegypti das populações de Campina Grande e Remígio.

Os efeitos da temperatura sobre o ciclo de vida de insetos vetores foram relatados por diversos autores (Costa et. al., 1994; RibeirRo et. al., 2001; CAlADO \& NAVARRO-SilVA, 2002a,b,c; BeserRa et. al., 2006). Estudos com Culex quiquefasciatus Say, 1823, demonstraram que ocorria desenvolvimento embrionário em temperaturas que oscilaram entre $10^{\circ} \mathrm{Ce} 34^{\circ} \mathrm{C}$, mas não a $5^{\circ} \mathrm{C}$ e a $40^{\circ} \mathrm{C}$, e o período de desenvolvimento larval diminuiu de 53,7 dias a $15^{\circ} \mathrm{C}$ para 7,4 dias a $30^{\circ} \mathrm{C}$ (CosTA et. al., 1994). Para essa espécie também foi constatada uma viabilidade dos ovos acima de $97,9 \%$ em temperaturas entre $15^{\circ} \mathrm{Ce} 30^{\circ} \mathrm{C}$ e uma diminuição no período de desenvolvimento larval de 35,7 dias a $15^{\circ} \mathrm{C}$ para 7,5 dias a $30^{\circ} \mathrm{C}$ (Ribeirro et. al., 2004). Contudo, deve-se ressaltar que temperaturas baixas geralmente são deletérias ao desenvolvimento dos insetos (e.g. A. albopictus, para o qual a temperatura de $20^{\circ} \mathrm{C}$ ocasionou $50 \%$ de mortalidades dos ovos) (CALADO \& NAVARRo-SILVA, 2002c). Para C. quinquefasciatus e A. aegypti foram registradas baixas infestações em consequência das baixas temperaturas do estado de São Paulo, provavelmente devido à menor fecundidade das fêmeas e ao aumento no período de desenvolvimento das fases imaturas (GLASSER \& GOMES, 2002). Em acréscimo, o maior tempo de desenvolvimento pode acarretar um aumento da mortalidade dos imaturos em condições naturais, dado o maior tempo de exposição à predação, parasitismo e doenças (TRIPS \& SHEMANChUK, 1970).

Conforme observado para as fases de desenvolvimento dos imaturos de A. aegypti, a temperatura teve efeito sobre a fase adulta e os extremos térmicos de $18^{\circ} \mathrm{C}$ e $34^{\circ} \mathrm{C}$ diminuíram a longevidade dos adultos e a fecundidade das fêmeas. Isto também foi constatado para populações de A. aegypti provenientes dos municípios de Boqueirão, Brejo dos Santos, Campina Grande, Itaporanga e Remígio (onde verificou-se menor longevidade dos adultos e menor fecundidade das fêmeas quando foram submetidas a temperaturas de $18^{\circ} \mathrm{Ce} 34^{\circ} \mathrm{C}$ ) (BESERRA et. al., 2006) e para A. albopictus cuja longevidade média foi de 19,4 e 13,6 dias para fêmeas e machos respectivamente (CALADO \& NAVARRo-Silva, 2002c).

Verificou-se que os limites térmicos (tb) de desenvolvimento e as respectivas constantes térmicas (K), expressas em graus-dias (GD), para as fases de ovo, larva, pupa e de ovo à emergência do adulto, variaram de acordo com os estágios de desenvolvimento e populações estudadas, o que também foi verificado para Ophyra aenescens Wiedemann 1830 (Diptera: Muscidae) (Ribeirro et. al., 2001), A. albopictus (CALADO \& NAVARROSiLVA, 2002b), C. quinquefasciatus (RIBEIRRo et. al., 2004) e A. aegypti (BESERRA et. al., 2006). Tais variações são consequências das diferenças estruturais e fisiológicas inerentes a cada estágio de desenvolvimento do inseto, que garante à espécie a capacidade de adaptação às variações ambientais (CALADO \& NAVARRO-SILVA, 2002b).

$\mathrm{Na}$ presente pesquisa constatou-se que a população de Remígio apresentou a maior temperatura base para o desenvolvimento do inseto de ovo à emergência do adulto, sendo as menores para a população de Campina Grande. Tal fator não era esperado por estarem esses dois municípios localizados em uma mesma região biogeográfica, nordestino quente de seca atenuada, com normais térmicas em média de $23,3^{\circ} \mathrm{C}$ (Laboratório de Meteorologia, Recursos Hídricos e Sensoriamento Remoto da Paraíba). Contudo, os resultados obtidos estão de acordo com estudos feitos com população de A. aegypti coletada em Remígio, para a qual o limite térmico inferior para o desenvolvimento de ovo a adulto foi de $13,5^{\circ} \mathrm{C}$, sendo este superior ao que foi constatado para outras populações como de Boqueirão $\left(9,5^{\circ} \mathrm{C}\right)$, Brejo dos Santos $\left(8,5^{\circ} \mathrm{C}\right)$, Itaporanga e Campina Grande $\left(3,5^{\circ} \mathrm{C}\right)$ (BESERRA et. al., 2006).

Com base no tempo de desenvolvimento e viabilidade das fases de ovo, larva e pupa e na fecundidade dos adultos, verificou-se que a temperatura favorável ao vetor encontra-se acima dos $22^{\circ} \mathrm{C}$ e abaixo dos $32^{\circ} \mathrm{C}$, portanto dentro da faixa de temperatura das suas regiões de ocorrências. As temperaturas bases para o desenvolvimento das diferentes fases do ciclo de vida do inseto foram inferiores às temperaturas mínimas de cada região. Assim, considerando-se a temperatura média ambiente de cada região onde estão situados os municípios de Boqueirão, Campina Grande e Remígio (aproximadamente $25^{\circ} \mathrm{C}$ ) todas as localidades apresentam condições favoráveis ao desenvolvimento e estabelecimento das populações de A. aegypti, podendo este apresentar acima de vinte gerações ao longo do ano. 
Agradecimentos. Ás Secretarias Municipais de Saúde dos Municípios de Boqueirão, Campina Grande e Remígio, Paraíba. À Organização Pan-Americana de Saúde e Ministério da Saúde pelo apoio financeiro através de carta acordo BR/LOA/0500008.002.

\section{REFERÊNCIAS BIBLIOGRÁFICAS}

Aldama, P. C. \& Garcia, F. J. H. 2001. Ciclo de vida del Aedes aegypti y manifestaciones clínicas del dengue. Acta Pediátrica de México 22(2):114-117.

Beserra, E. B; Castro-Júnior, F. P; Santos, J. W; Santos, T. S. \& FERnANDES, C. R. M. 2006. Biologia e exigências térmicas de Aedes (Stegomyia) aegypti (Diptera: Culicidae) provenientes de quatro regiões bioclimáticas da Paraíba. Neotropical Entomology 35(6):853-860.

Calado, D. C. \& Navarro-Silva, M. A. 2002a. Avaliação da influência da temperatura sobre o desenvolvimento de Aedes albopictus. Revista de Saúde Pública 36(2):173-179.

2002b. Exigências térmicas de Aedes (Stegomyia) albopictus Skuse, 1894 (Diptera, Culicidae) em condições de laboratório. Revista Brasileira de Entomologia 46(4):547-551.

2002c. Influência da temperatura sobre a longevidade, fecundidade e atividade hematofágica de Aedes (stegomyia) albopictus Skuse, 1894 (Diptera, Culicidae) sob condições de laboratório. Revista Brasileira de Entomologia 46(1):93-98.

Costa, P. R. P.; Viana, E. E. S.; Silveira, Jr. P. \& Ribeiro, P. B. 1994. Influência da temperatura na longevidade e viabilidade do ciclo aquático do Culex quinquefasciatus Say, 1823 (Diptera: Culicidae) em condições de laboratório. Revista Brasileira de Parasitologia Veterinária 3(2):87-92.

Forattini, O. P. 2002. Culicidologia Médica. São Paulo, Edusp. $857 \mathrm{p}$.
Glasser, C. M. \& Gomes, A. C. 2002. Clima e sobreposição da distribuição de Aedes aegypti e Aedes albopictus na infestação do Estado de São Paulo. Revista de Saúde Pública 36(2):166-172.

Haddad, M. L. \& Parra, J. R. P. 1984. Métodos para estimar os limites térmicos e a faixa ótima de desenvolvimento das diferentes fases do ciclo evolutivo dos insetos. Piracicaba, FEALQ. 12p.

Honório, N. A. \& Lourenço-De-Oliveira, R. 2001. Frequência de larvas e pupas de Aedes aegypti e Aedes albopictus em armadilha, Brasil. Revista de Saúde Pública 35(4):385-391.

Lozovei, A. L. 2001. Culicídeos (mosquitos). In: Marcondes, C. B. eds. Entomologia Médica e Veterinária. São Paulo, Atheneu. p.59-104.

Natal, D. 2002. Bioecologia do Aedes aegypti. Biológico 64(2):205-207.

Ribeiro, P. B.; Carvalho, C. J. B.; Regis, M. \& Costa, P. R. P. 2001 Exigências térmicas e estimativa do número de gerações de Ophyra aenescens Wiedemann, 1830 (Diptera, Muscidae, Azeliinae), em Pelotas, RS. Arquivo do Instituto Biológico 68(1):75-82.

Ribeiro, P. B; Costa, P. R. P; Loeck, A. E; Viana, E. E. S. \& Silveira JúNIOR, P. 2004. Exigências térmicas de Culex quinquefasciatus (Diptera, Culicidae) em Pelotas, Rio Grande do Sul, Brasil. Iheringia, Série Zoologia, 94:177-180.

Silveira-Neto, S.; Nakano, O.; Barbin, D. \& Villa Nova, N. 1976. Manual de ecologia dos insetos. São Paulo, Agronômica Ceres. $419 \mathrm{p}$.

Trips, M. \& Shemanchuk, J. A. 1970. Effect of constants temperature on the larval development of Aedes vexans (Diptera: Culicidae). The Canadian Entomologist 102: 1048-1051. 